\title{
First exoplanet found around a Sun-like star
}

\section{Eliza Kempton}

In 1995, astronomers detected a blisteringly hot Jupiter-mass planet orbiting closer to its host star than Mercury is to the Sun. This discovery recast our thinking of how planets form and led to a new era of exoplanetary exploration.

Anyone over the age of 35 will remember growing up in a world in which only one planetary system was known - our own. We remember proudly reciting the names of the nine planets (eightbefore Pluto's discoveryin1930, and again today with its reclassification as a dwarf planet in 2006) and wondering what other planets might exist around the stars in the night sky. Contemplating life beyond the Solar System was relegated to science fiction. This all changed in 1995 when Mayor and Queloz ${ }^{1}$ reported the detection of the first exoplanet around a Sun-like star.

The discovery of the gas-giant planet named 51 Pegasi b after its parent star, 51 Pegasi - came as a surprise. Gas-giant planets, such as Jupiter, are located in the outer parts of the Solar System. The prevailing theory was, and still is, that the formation of these planets requires icy building blocks that are available only in cold regions far away from stars. Yet Mayor and Queloz found 51 Pegasi b to be orbiting about ten times closer to its host star than Mercury is to the Sun (Fig. 1). One possible explanation is that the planet formed farther out and then migrated to its current location.

The gas-giant planet was not the first exoplanet to be discovered. However, the previous detections ${ }^{2,3}$ were of even stranger objects orbiting pulsars - rapidly spinning neutron stars, which are the collapsed remnants of hot massive stars. The discovery of 51 Pegasi b was the first to substantiate the existence of planets around long-lived hydrogen-burning stars that resemble the Sun.

The bizarre character of a gas-giant planet orbiting so close to its parent star engendered considerable scepticism about the true nature of 51 Pegasi b. Mayor and Queloz detected the planet through minute back-and-forth motion of 51 Pegasi, which seemed to indicate that a planet-mass object was pulling on the star. But this stellar motion, sensed by frequency shifts in the spectra of light from 51 Pegasi, had other possible interpretations. A lively debate ensued in the literature about whether pulsations of the star might be masquerading as a planetary signature $\mathrm{e}^{4,5}$.
This debate was put to rest in 1998 when the astronomer David F. Gray wrote a paper refuting his previous assertion that the stellar spectra were indicative of pulsations rather than a planet ${ }^{6}$. Further vindication came through the detection of planets similar to 51 Pegasi b, as other researchers combed their existing data for similarly unexpected planetary signals ${ }^{7}$. These highly irradiated giant planets have come to be known as hot Jupiters.

In the 24 years since the discovery of 51 Pegasi b, about 4,000 exoplanets have been identified (see go.nature.com/2jpcgtf). Other detection techniques have entered the scene, including the transit method, in which an exoplanet is revealed through the subtle dimming of its host star as the planet crosses the line of sight between Earth and the star. Hot Jupiters have continued to be discovered by the many exoplanet searches that are sensitive to large planets on close orbits. However, it is now known that such objects are intrinsically rare, orbiting only about $1 \%$ of Sun-like stars ${ }^{8}$.
By contrast, planets known as super-Earths and mini-Neptunes abound. Such objects, which inhabit the size and mass gap between the rocky and gas-giant planets of the Solar System, were also a surprise to planet hunters, but seem to be commonplace in our Galaxy. There is now good reason to think that the Milky Way contains more planets than it does stars?.

Mayor and Queloz's detection of 51 Pegasi b gave rise to a new field of astronomy. The ranks of exoplanet researchers have been steadily growing, by some counts now making up about one-quarter of the astronomy profession (see go.nature.com/32imc4j). Incipient subfields include the study of exoplanet demographics and the characterization of exoplanetary atmospheres.

This characterization has confirmed that hot Jupiters truly are gas-giant planets, but ones representing what our own Jupiter would look like if it were suddenly transported 100 times closer to the Sun. Amid the scorching-hot hydrogen-helium envelopes of these planets, astronomers have detected trace amounts of steam, carbon monoxide and metal vapours ${ }^{10-12}$. Such atmospheric studies could lead to the eventual characterization of exoplanets that resemble Earth.

The future of the exoplanet field is bright. In April 2018, NASA launched the Transiting Exoplanet Survey Satellite (TESS), a space telescope that is just beginning to fulfil its mission of finding small transiting planets around the brightest stars in the night sky. These planets will be ideally suited for follow-up using NASA's James Webb Space Telescope (JWST), once it launches, to measure their atmospheric properties and compositions. Following on the heels of JWST, the European Space Agency has selected the Atmospheric Remote-sensing Infrared Exoplanet Large-survey (ARIEL) space

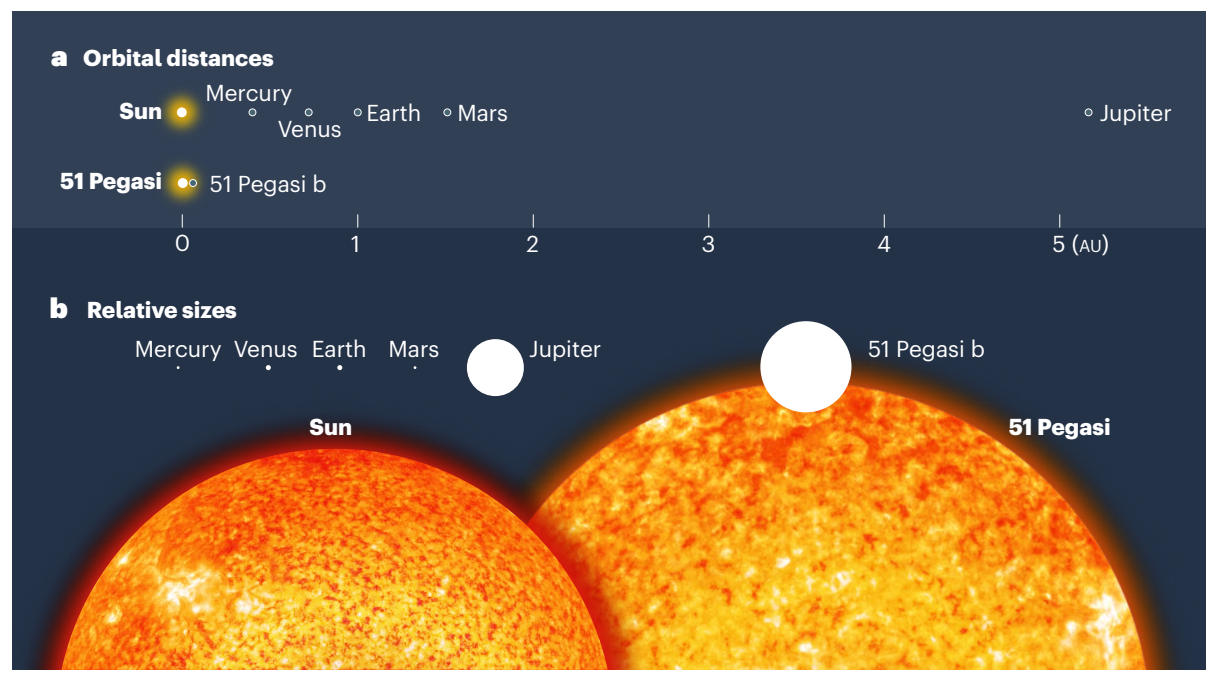

Figure 1 | The planetary systems of the Sun and of 51 Pegasi. a, In the Solar System, gas-giant planets, such as Jupiter, orbit far from the Sun. In 1995, Mayor and Queloz ${ }^{1}$ reported the discovery of 51 Pegasi b - a gasgiant planet that is much closer to its host star, 51 Pegasi, than Mercury is to the Sun. The orbital distances of the planets are given in astronomical units ( $1 \mathrm{AU}$ is the average separation between Earth and the Sun). b, The sizes of all objects are shown approximately to scale. 


\section{0 extraordinary papers}

telescope to launch in 2028. ARIEL will be dedicated to characterizing the atmospheres of a wide sample of exoplanets.

These programmes are paving the way towards the ultimate goal of potentially detecting the signatures of life on an exoplanet. This goal could most optimistically be achievable in the next decade, but more realistically will require a new generation of space- and ground-based telescopes ${ }^{13}$. What is remarkable is that humans have gone from discovering the first exoplanets to legitimately plotting out the search for life on these worlds in just a quarter of a century.

Eliza Kempton is in the Department of Astronomy, University of Maryland,
College Park, Maryland 20742, USA.

e-mail: ekempton@astro.umd.edu

1. Mayor, M. \& Queloz, D. Nature 378, 355-359 (1995).

2. Wolszczan, A. \& Frail, D. A. Nature 355, 145-147 (1992).

3. Wolszczan, A. Science 264, 538-542 (1994).

4. Gray, D. F. Nature 385, 795-796 (1997).

5. Hatzes, A. P., Cochran, W. D. \& Johns-Krull, C. M. Astrophys. J. 478, 374-380 (1997).

6. Gray, D. F. Nature 391, 153-154 (1998).

7. Butler, R. P., Marcy, G. W., Williams, E., Hauser, H. \& Shirts, P. Astrophys. J. 474, L115-L118 (1997).

8. Howard, A. W. et al. Astrophys. J. Suppl. 201, 15 (2012).

9. Batalha, N. M. Proc. Natl. Acad. Sci. USA 111, 12647-12654 (2014).

10. Kreidberg, L. et al. Astrophys. J. 793, L27 (2014)

11. Snellen, I. A. G., de Kok, R. J., de Mooij, E. J. W. \& Albrecht, S. Nature 465, 1049-1051 (2010).

12. Hoeijmakers, H. J. et al. Nature 560, 453-455 (2018).

13. National Academies of Sciences, Engineering, and Medicine. Exoplanet Science Strategy (National Academies, 2018).

\section{Cell biology}

\section{Cell identity reprogrammed}

\section{Samantha A. Morris}

The discovery that cell differentiation can be reversed challenged theories of how cell identity is determined, laying the foundations for modern methods of reprogramming cell identity and promising new regenerative therapies.

All cells of an organism derive from a single cell. As development progresses, cells become increasingly specialized to perform defined functions, a commitment that is accompanied by a restriction in the range of potential fates of those cells. In the late nineteenth century, a predominant thought was that, when they differentiate, cells retain only those pieces of heritable information required to maintain cell-type identity and function ${ }^{1}$. This led to the theory that differentiation is an irreversible process (Fig.1a). John Gurdon's seminal paper in Nature on nuclear reprogramming of cell identity, with Tom Elsdale and Michael Fischberg ${ }^{2}$, provided a remarkable challenge to this dogma, and formed the basis for today's cell-reprogramming field.

Gurdon and colleagues' 1958 paper was preceded by the work of Robert Briggs and Thomas King ${ }^{3}$. To investigate the developmental potential of differentiating cells, Briggs and King used a method called nuclear transfer, in which the nucleus is removed from one cell (in this case, an egg) and replaced with an intact nucleus from a different cell. Briggs and King's experiments were a technical feat that had previously been accomplished only in single-celled organisms ${ }^{4}$.

Using this method in the more-complex Northern leopard frog (Rana pipiens), they were able to produce normal, swimming tadpoles by replacing egg-cell nuclei with nuclei from blastomeres - cells that are made through the splitting of a fertilized egg cell during early development ${ }^{3}$. However, the transfer of nuclei from $R$. pipiens cells at more-advanced stages of differentiation from when the hollow ball of blastomeres differentiates into a multilayered structure called a gastrula, onwards - did not support the development of normal frogs ${ }^{5}$ (Fig. 1b).

\section{"Since this paper appeared, biologists have developed the ability to reprogram cell identity by several routes."}

Thus, Briggs and King's results demonstrated that the nuclei in blastomeres are not irreversibly changed with differentiation. However, they also indicated that, as development progresses, the potential of transplanted nuclei to support normal development decreases suggesting that cell differentiation might be irreversible and might involve irreversible genetic changes. Thus, Briggs and King concluded $^{5}$ that the nuclei of cells in the latestage gastrula have an "intrinsic restriction in potentiality for differentiation".

In 1958, Gurdon, Elsdale and Fischberg addressed the questions surrounding the potential of differentiated cells using a different species of frog,Xenopus laevis (the African clawed frog). In contrast to the Rana species, whose availability is seasonally restricted, $X$. laevis is available year round and rapidly reaches sexual maturity ${ }^{2}$. In the authors' experiments, donor nuclei from cells at various developmental stages, from early blastomeres to cells from tadpoles just before hatching, were transferred into Xenopus egg cells.

The donor nuclei were derived from a mutant stock in which each cell contained only one nucleolus (an organelle inside the nucleus) instead of the usual two. This approach provided a useful visual marker to confirm that the resulting animals obtained from nuclear transfer were indeed derived from the transferred nucleus, and not from existing material in the egg. These experiments demonstrated that normal tadpoles could be obtained from cells at stages of development up to pre-hatching tadpole stages (Fig. 1c) - much later than the developmental stage of the cells that Briggs and King had used.

Many of the tadpoles that developed from cells containing transferred nuclei underwent normal metamorphosis into frogs, which seemed to be sexually mature. The authors noted that the lone frog derived from the most-differentiated cell nucleus was "accidentally killed shortly before metamorphosis". A subsequent report ${ }^{6}$ was free of such misadventure; it described the derivation of fertile adult frogs from the transplanted nuclei of fully differentiated cells collected from the intestines of feeding tadpoles.

Gurdon and colleagues thus demonstrated, unlike Briggs and King, that differentiated nuclei could support successful development. Despite this discordance, both groups agreed that the advance of a nucleus through differentiation was accompanied by a reduction in its ability to support normal development. On the basis of their findings that some differentiated nuclei could support normal development (albeit with a relatively limited frequency of success), Gurdon and colleagues concluded that the differentiated cell state is not a result of irreversible genomic changes. Rather, the nuclei of differentiated cells retain the capacity to orchestrate the development of a fully functioning organism.

Almost 40 years after these amphibian experiments, transfer of the nucleus of an adult mammary epithelial cell was used to generate a cloned mammal: Dolly the sheep ${ }^{7}$. The first mouse to be cloned using nuclear transfer from adult cells, Cumulina, was reported shortly afterwards ${ }^{8}$. To prove beyond doubt that cloned animals could be produced using nuclei from fully differentiated cells (and had not previously been derived from contaminant 